\title{
A fresh approach to interpretation of visible photoluminescence spectra in silicon nanostructures
}

\author{
A.V. Sachenko, Yu.V. Kryuchenko, E.G. Manoilov, E.B. Kaganovich \\ V. Lashkaryov Institute of Semiconductor Physics NAS of Ukraine, 41, prospect Nauky, 03028 Kyiv, Ukraine \\ Phone:+380(44)2656391,E-mail:div47@isp.kiev.ua
}

\begin{abstract}
To understand both multimodal character of stationary photoluminescence (PL) spectra and observed peculiarities in time-resolved PL in low-dimensional Si structures, it is proposed to take into account an additional effect, which has to emerge in such structures due to indirect-bandgap nature of silicon material. The effect implies that the exciton radiative lifetime becomes a nonmonotonous (oscillating) function of the nanocrystal (NC) size. As a result, in the calculated PL spectra the energy distance between PL peaks or PL minima practically determined by the mean NC size, while dispersion in NC sizes plays a minor role. The qualitative agreement between calculated PL spectra and PL spectra observed experimentally in porous silicon and nanocrystalline silicon (nc-Si) films counts in favor of the used model of radiative exciton recombination.
\end{abstract}

Keywords: exciton transitions, silicon nanocrystals, quantum size effect, radiative recombination time, photoluminescence, time-resolved spectroscopy.

Paper received 10.11.03; accepted for publication 11.12.03.

\section{Introduction}

As shown in [1], the probability of quasi-direct (i.e. without phonon assistance) excitonic radiative transitions in nc-Si structures is an oscillating function of NC characteristic size $D$. This effect is simply a result of oscillations in the overlap integral (entering into the matrix element of such transitions) taken on the confined electron and hole envelope wave functions originating from conduction band $X$-valleys and valence band $\Gamma$-valley of bulk silicon, respectively. These oscillations in turn arise due to remaining contributions $\sim \exp \left(i K_{0} r\right)$ into the envelope electron wave function from $X$-valley minima at the points like $K_{0}=0.85(2 \pi / a)[1,0,0]$, i.e. due to indirect-bandgap structure of silicon material. Simple estimation of the period of such oscillations in silicon nanostructures gives the value of about $0.64 \mathrm{~nm}$. Corresponding theoretical calculations of characteristic times $\tau_{r x}^{d}(D)$ for such radiative quasi-direct exciton transitions in $\mathrm{Si}_{-} \mathrm{SiO}_{\mathrm{x}}$ quantum wires $(\mathrm{QW})$ have been made in [2]. Results of these calculations are shown in the inset in Fig. 1. As one can see from this figure, the increase of the oxygen content $x$ in the barrier material leads to a shift of oscillating $\tau_{r x}^{d}(D)$ - dependencies towards larger $D$ values. A substantial overall decrease of the exciton radiative lifetime with wire thickness decrease occurs due to stronger localization of carriers in narrower QWs, or, in other words, due to increasing probability for $X$-valley electrons to give up the excess pulse directly to a nanostructure as a whole and take part in the recombination process without phonon assistance. A goal of the present work is to give an analysis of the features, which $\tau_{r x}^{d}(D)$-oscillations can bring into PL spectra of silicon nanostructures, and to interpret experimentally observed PL spectra from this point of view.

\section{Theoretical and experimental results}

In the following theoretical analysis we restrict our consideration to the range of QW thicknesses small enough to provide sufficiently large binding energies of the exciton states $(>0.3 \mathrm{eV})$ in $\mathrm{QWs}$, when contribution into room-temperature PL of free electron-hole pairs can be neglected. In this case the generation-recombination balance equation for an isolated QW of a diameter $D$ takes the following form: 
$\frac{d n_{x}(D)}{d t}+\frac{n_{x}(D)}{\tau_{x}(D)}=c D^{2} I[1-\exp (-\alpha(D) L)]$

where $n_{x}(D)$ is the total number of excitons in QW (exciton concentration integrated over QW length $L$ ) in the lowest exciton sub-band, $I$ is the intensity of laser excitation, $\alpha(D)$ is the absorption coefficient, $c \sim 1$ is a form-factor ( $c=1$ for QWs having quadratic cross-section and $c=\pi / 4$ for cylindrical QWs $), \tau_{x}(D)$ is the total lifetime of excitons in $\mathrm{QW}, 1 / \tau_{x}(D)=1 / \tau_{r x}^{d}(D)+1 / \tau_{r x}^{i}(D)+1 / \tau_{n x}(D)$, where $\tau_{n x}(D)$ is the characteristic time of nonradiative exciton recombination in $\mathrm{QW}$, while $\tau_{r x}^{d}(D)$ and $\tau_{r x}^{i}(D)$ are the characteristic times of radiative quasi-direct (without phonon assistance) and indirect (with phonon assistance) exciton transitions in QW, respectively. As follows from (1), at stationary conditions $n_{x}(D)$ is expressed as

$$
n_{x}(D)=c D^{2} I[1-\exp (-\alpha(D) L)] \tau_{x}(D)
$$

while the intensity of the exciton PL-line at the corresponding energy $E(D)$ of the exciton transition equals to

$$
\mathfrak{I}_{P L}(E)=c D^{2} I[1-\exp (-\alpha(D) L)] \tau_{x}(D) / \tau_{r x}(D),
$$

where $\tau_{r x}(D)$ is the total characteristic time of radiative exciton transition, which is determined by the following reciprocal law relation: $1 / \tau_{r x}(D)=1 / \tau_{r x}^{i}(D)+1 / \tau_{r x}^{d}(D)$.

For an ensemble of quantum wires with their thickness distribution described, for instance, by Gauss function $f_{G}(D, \bar{D}, \sigma)$, where $\bar{D}$ is the most probable thickness and $\sigma$ is the QW thickness dispersion, the spectral density of the exciton PL is expressed as

$$
I_{P L}(E)=\mathfrak{I}_{P L}(E) f_{G}(D(E), \bar{D}, \sigma) \frac{\partial D}{\partial E}
$$

where $D(E)$ is the reverse function to the $E(D)$-dependence for the energy of the exciton transition obtained earlier in [1].

The kinetics of PL-line intensity decay at an energy $E(D)$ after switching off a short (relatively to the characteristic time $\left.\tau_{x}(D)\right)$ exciting pulse is determined by the following expression:

$$
\begin{aligned}
& \mathfrak{I}_{P L}(t, E)=c D^{2} I[1-\exp (-\alpha(D) L)] \times \\
& \times \exp \left(-t / \tau_{x}(D)\right) t_{i} / \tau_{r x}(D),
\end{aligned}
$$

where $t_{i}$ is the laser pulse duration.

Assuming the Gauss form for QWs size distribution the kinetics of the integral PL intensity decay in corresponding nanostructures can be expressed as follows:
From the fact that integral exciton PL is determined by the contributions from QWs having different thicknesses in the range from $D_{\text {min }}$ to $D_{\max }$, and characteristic times of the exciton recombination are nonmonotonous (oscillating) functions of QW thicknesses in this range, it follows that relaxation of the exciton PL spectrum has to be rather complex function of the time, which doesn't correspond to piecewise exponential dependence. Even in a case of small quantum efficiencies of exciton PL, when $\tau_{r x} \gg \tau_{n x}$ and $\tau_{r x}^{d}(D)$-oscillations don't manifest themselves in PL relaxation times, they can effect substantially on the PL intensity amplitude. Leaving a detailed analysis of the time dependencies of the integral PL for the future, here we focus our attention only on the influence of oscillating $\tau_{r x}^{d}(D)$-dependencies on PL spectra.

Calculating stationary PL spectra we have used $\tau_{r x}^{d}(D)$-dependencies obtained in [2] for the case of oxygen content $x=2$ in the barrier material $\mathrm{SiO}_{x}$ around silicon QWs and corresponding $E(D)$-dependencies for the energy of the exciton radiative transition obtained in [1]. A case of strong absorption, when $\alpha(D) L>>1$, has been a subject of interest.

If size distribution of QWs is described by a sum of several Gauss functions $f_{G}\left(D, \bar{D}_{i}, \sigma\right)$ with several $\bar{D}_{i}$ values, $i=1,2,3 \ldots$, then in the case of small $\mathrm{QW}$ thickness dispersion around $\bar{D}_{i}$ values (e.g. when $\sigma=0.1 \mathrm{~nm}$ ) the stationary PL spectrum consists of relatively narrow bands, each isolated PL band corresponding to a particular Gauss function with a particular $\bar{D}_{i}$ value and particular weight coefficient $a_{i}$. With $\sigma$ increase the PL bands become wider and overlap one another giving a structured total PL spectrum. In practice, it is a standard way of obtaining and explanation of structured PL spectra. However, according to our model, even for a single Gauss distribution (e.g. for a single $\bar{D}$ value of about 2 $\mathrm{nm}$ ) it is possible to obtain several (up to three depending on $\sigma$ value) PL bands in the vicinity of the energies corresponding to the minima in oscillating $\tau_{r x}^{d}(D)$-dependencies. In a total, varying mean $\mathrm{QW}$ thickness $\vec{D}$ within $1.2-3 \mathrm{~nm}$ range we can obtain in this way up to five PL bands for the cases of QWs of both quadratic and round cross-sections with PL band maxima lying within 1.131.14 ; $1.17-1.21,1.24-1.34,1.45-1.62$ and $1.93-2.26 \mathrm{eV}$ energy ranges.

Note, that analogous effect can be responsible also for the multimodal character of the PL from silicon quantum $\operatorname{dot}(\mathrm{QD})$ nanostructures, i.e. as in QW-case the main reason of structured QD PL spectra can be connected with the existence of several minima in oscillating $\tau_{r x}^{d}(D)$-dependencies which appear due to indirect-bandgap nature of silicon material. Quantitative (but not qualitative) discrepancy between PL spectra of silicon QDs and QWs consists in the energy shift of QD PL spectra into the region of

$$
I_{\text {int }}^{P L}(t)=c \int_{D_{\min }}^{D_{\max }}\left(\xi^{2} I[1-\exp (-\alpha(\xi) L)] \exp \left(-t / \tau_{x}(\xi)\right) t_{i} / \tau_{r x}(\xi)\right) f_{G}(\xi, \bar{D}, \sigma) \partial \xi
$$




\section{A.V. Sachenko et al.: A fresh approach to interpretation of visible photoluminescence...}

shorter wavelengths. Estimations which we have made for silicon QD nanostructures exploiting both $i$ ) size dependencies of the energies of one-particle (electron and hole) excitations in QDs as well as of the binding energies of electron-hole pairs (see $[3,4]$ ) and $i$ ) size dependencies of the energies of exciton transitions in QWs [1] multiplied by a factor of about 1.3 have shown that in the case of silicon QDs in $\mathrm{SiO}_{2}$ matrix five PL bands within 1.26$1.40,1.42-1.59,1.68-1.80,2.08-2.14$ and $2.57-2.79 \mathrm{eV}$ energy ranges have to be observed in QD PL spectra.

Fig.1 shows two calculated spectra of a stationary PL in silicon QW structure. Curve 1 is built with an account of quasi-direct radiative exciton transitions in QWs while curve 2 without such an account. Both curves are built using the Gauss size distribution of QWs with the large dispersion parameter $\sigma=0.5 \mathrm{~nm}$. As one can see from this figure, in the first case (curve 1) PL spectrum contains three bands which arise due to oscillating character of $\tau_{r x}^{d}(D)$-dependence discussed above, while PL spectrum illustrated by the curve 2 consists only of one band.

Fig. 2 demonstrates the time-resolved PL spectra of porous silicon measured in the experiment [5]. A special features of these spectra are the existence of at least two PL bands and their low-energy shift with delay time $t_{d}$. To our mind, such a shift can be explained by faster radiative recombination in QWs which thiknesses correspond to smaller $\tau_{r x}^{d}$ values. Indeed, the narrower are the QWs the smaller in average are the respective times of

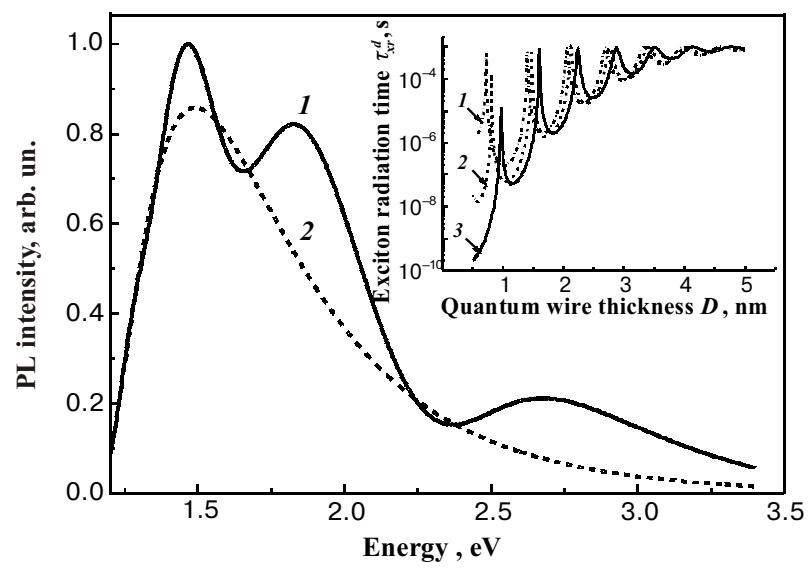

Fig. 1. Calculated spectra of stationary PL for silicon QW structures. Curve 1 is obtained with an account for quasi-direct (i.e. without phonon assistance) radiative exciton transitions characterized by oscillating $\tau_{r x}^{d}(D)$-dependence, while curve 2 without such an account. Parameters of calculation: mean QW thickness $\bar{D}=2 \mathrm{~nm}$, thickness dispersion $\sigma=0.5 \mathrm{~nm}$, nonradiative exciton lifetime for QW of the diameter $D=3 \mathrm{~nm} \tau_{n x}=1 \mathrm{mcs}$, characteristic time of radiative indirect exciton transition for QW of the diameter $D=3 \mathrm{~nm} \tau_{r x}^{i}=20 \mathrm{mcs}$. The inset presents $\tau_{r x}^{d}(D)$-curves corresponding to different oxygen content $x$ in $\mathrm{SiO}_{\mathrm{x}}$ barrier material: $x=1.5(1), x=1.75(2), x=2$ (3).

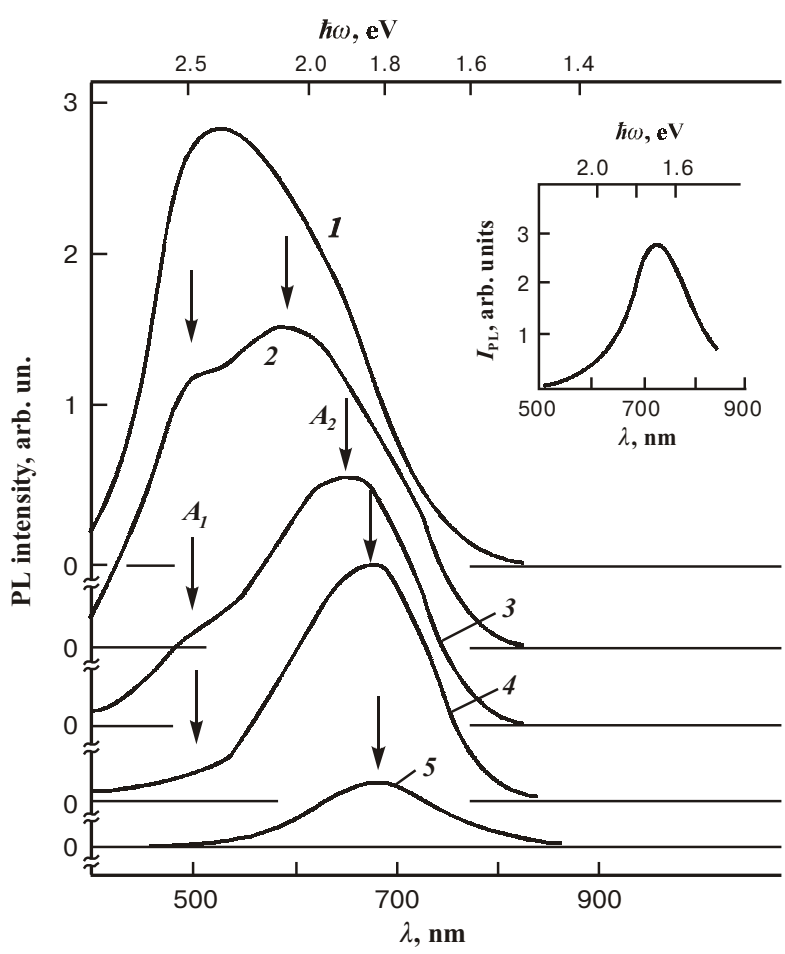

Fig. 2. Evolution of PL spectra fixed by the method of time-resolved spectroscopy. Delay times $t_{d}$, ns: 10(1), 25(2), 50(3), 100(4), 1000(5). The spectrum of stationary PL is shown in the inset [5].

quasi-direct radiative recombination of excitons and higher are the energies of exciton transitions.

The above discussed features of our approach are confirmed by the calculated time-resolved spectra of the exciton PL in silicon QW structure, see Fig. $3 a$. These spectra have been obtained by assuming that distribution of QWs over their diameters is described by a sum of several Gauss functions with different $\vec{D}_{i}$ values, $i=1,2,3 \ldots n$ and different weight coefficients $a_{i}$ :

$$
I_{P L}\left(t_{d}, E\right)=\sum_{i=1}^{n} a_{i} \mathfrak{I}_{P L}\left(t_{d}, E\right) f_{G}\left(D(E), \bar{D}_{i}, \sigma\right) \frac{\partial D}{\partial E},
$$

It follows from a comparison of the Fig. 2 and Fig.3a spectra that within our model we can force both energy position of PL bands and low-energy temporal shift of PL spectra to fit approximately the experimental data. Moreover, maximum in the calculated stationary PL spectrum also corresponds to the observed in experiment.

At the same time, an analysis has shown that PL bands shift during PL relaxation occurs only if the maximum in Gauss size distribution of QWs doesn't correspond to the energy position of the PL bands. If such a coincidence takes place then only relative changes in the intensities of PL bands occur with the time, while the energy position of corresponding PL bands remains unchanged, see Fig. 3b. 


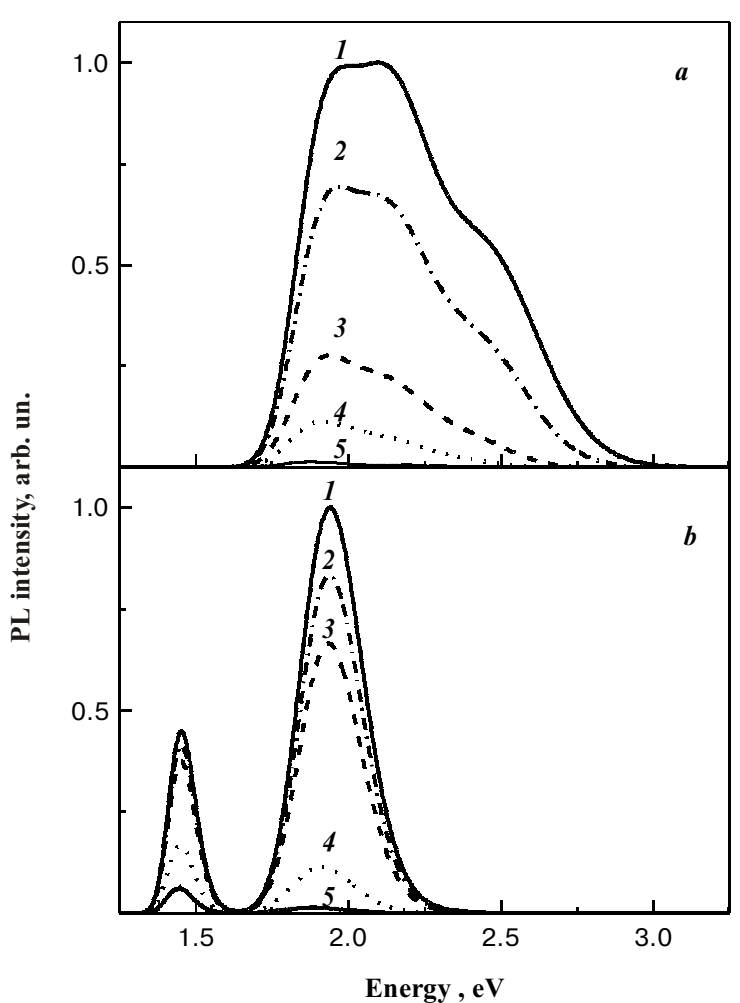

Fig. 3. Exciton PL spectra evolution in silicon QW nanostructures calculated in accordance with Eq.(7) for the case of QW size dispersion $\sigma=0.1 \mathrm{~nm}$. QW size distribution is described by a sum of (a) three Gauss functions with the parameters $\bar{D}_{1}=1.29 \mathrm{~nm}$, $\bar{D}_{2}=1.38 \mathrm{~nm}, \bar{D}_{3}=1.57 \mathrm{~nm}$ and weight coefficients $a_{1}=1.15$, $a_{2}=0.6, a_{3}=1.0$. Delay times $t_{d}$, ns: 25 (1), 100 (2), 300 (3), 500 (4), 1000 (5); (b) two Gauss functions with the parameters $\bar{D}_{1}=1.55 \mathrm{~nm}, \bar{D}_{2}=2.2 \mathrm{~nm}$ and weight coefficients $a_{1}=1.0$, $a_{2}=1.0$. Delay times $t_{d}$, ns: 10 (1), 50 (2), 100 (3), 500 (4), $1000(5)$.

Experimentally observed PL spectra of nc-Si films are shown in Fig. $4 a$ (stationary PL) and Fig. 4b (time-resolved PL). The films containing silicon QDs in $\mathrm{SiO}_{x}$ matrix have been obtained by pulsed laser deposition [6]. It follows from this figure, that both in stationary and time-resolved PL spectra several bands are observed. A study of film structure gave evidence of a wide dispersion of NC sizes, but no gaps in the QD size distribution $N(D)$ has been observed. Stationary PL spectra shown in Fig.4a correspond to the five different films with different passivation degree of the Si unsaturated dangling bonds (DB). Two PL bands with maxima at 1.59 and $2.35-2.5 \mathrm{eV}$ are clearly seen in these spectra. Intensity ratio for these two bands is determined by a competition between radiative and nonradiative channels of recombination in silicon NCs of different sizes. Nonradiative channel is connected with the existence of Si DB in NCs of the larger sizes. An analysis of the time-resolved PL spectra of the film, which stationary PL spectrum is shown

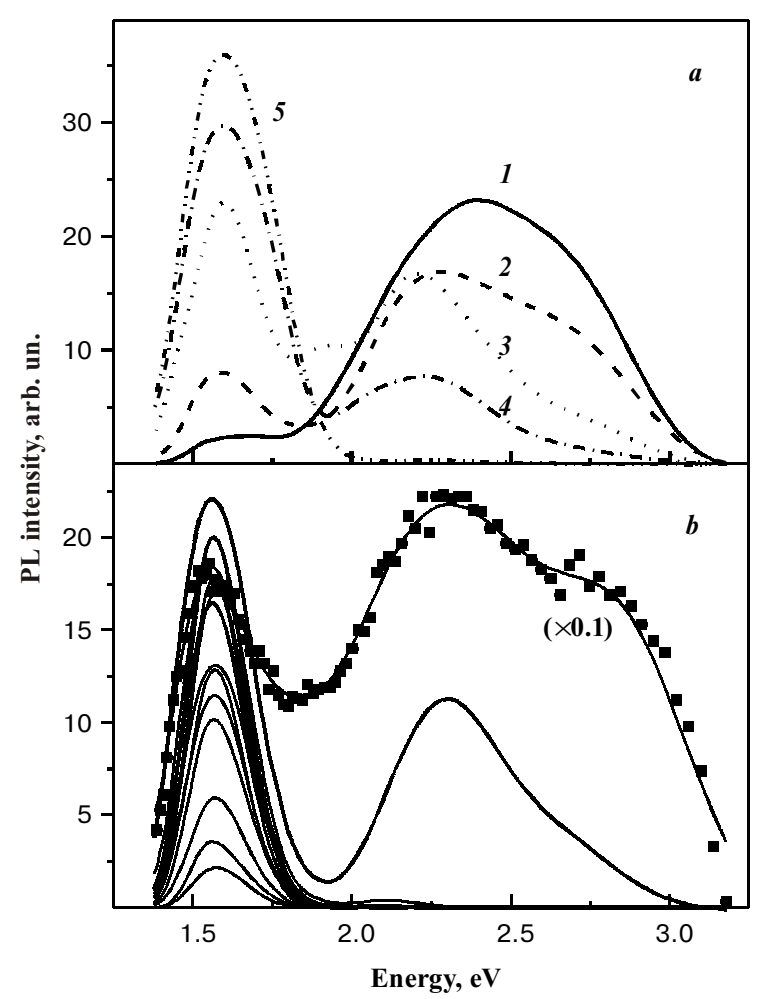

Fig. 4. PL spectra of nc-Si films obtained by pulsed laser deposition. (a) Stationary PL spectra of 5 different films with a decreased rate of nonradiative recombination occurring due to existence of Si DB in large-size silicon NC; $(b)$ time-resolved PL spectra of nc-Si film (stationary PL spectrum of which is given by the curve 2 in Fig. $4 a)$ in the delay time range from $250 \mathrm{~ns}(\times 0.1)$ to $20 \mathrm{mcs}$.

by the curve 2 in Fig.4a, gives evidence that at relaxation times up to $250 \mathrm{~ns}$ they contain four PL bands with the energies 1.56, 2.20, 2.58 and $2.91 \mathrm{eV}$ (Fig.4b). With delay time increase to $20 \mathrm{mcs}$ only one PL band at 1.56 $\mathrm{eV}$ is left in the PL spectrum. Note, that in the case of silicon QD films a number of bands in experimentally observed PL spectra is close to that predicted by theory, but exact fitting of energy positions of this bands really requires knowledge of many structure parameters and need further efforts in theoretical consideration. Nevertheless, we can conclude that in the case of nc-Si films a presence of several bands in PL spectra cannot be attributed to the existence of several preferable sizes of silicon $\mathrm{NCs}$ in the films.

Thus, the above made analysis enables us to associate multimodal character of stationary and time-resolved PL spectra in silicon NC structures with quantum oscillations in the size dependence of the exciton radiative recombination characteristic time. 


\section{A.V. Sachenko et al.: A fresh approach to interpretation of visible photoluminescence...}

\section{Acknowledgements}

The work was supported by INTAS grant (INTAS Call 2001 NANO-0444) and State Fundamental Research Foundation of Ukraine.

\section{References}

1. Yu.V. Kryuchenko, A.V. Sachenko, Quantum efficiency of exciton luminescence in low-dimensional structures with indirect energy gap // Physica E, 14, pp. 299-312 (2002).

2. D.V. Korbutyak, Yu.V. Kryuchenko, I.M. Kupchak, A.V. Sachenko, Characteristics of confined exciton states in silicon quantum wires // Semiconductor Physics, Quantum Electronics and Optoelectronics, 6(2), pp. 172-182 (2003).
3. M.V. Wolkin, J. Jorne, P.M. Fauchet, G. Allan and C. Delerue, Electronic states and luminescence in porous silicon quantum dots: The role of oxygen // Phys. Rev. Lett., 82(1), pp.197-200 (1999).

4. T. Takagahara, Effects of dielectric confinement and electron-hole exchange interaction on excitonic states in semiconductor quantum dots // Phys. Rev., B47(8), pp.4569-4584 (1993).

5. A.V. Andrianov, D.I. Kovalev, V.B. Sherman, I.D. Yarosheckii, Shortliving green band and time evolution of PL spectra in porous Si // Pis'ma JETF, 56, p. 242-245 (1992) (in Russian).

6. E.B. Kaganovich, E.G. Manoilov, I.R. Bazylyuk, S.V. Svechnikov, PL spectra of nanocrystalline silicon // Fiz. Tekh. Poluprovodn., 37(3), pp. 353-357 (2003) (in Russian). 